Tôhoku Math. Journ.

39 (1987), 575-588.

\title{
SPECTRAL GEOMETRY OF MINIMAL SURFACES IN THE SPHERE
}

\author{
Dedicated to Professor Luis Esteban Carrasco on his sixty-fifth birthday
}

Manuel Barros* and Francisco Urbano

(Received September 19, 1986)

Introduction. Let $M$ be a compact, connected, Riemannian manifold and $f \in C^{\infty}(M)$ (a smooth function on $M$ ). Then we have a spectral decomposition of $f$, say $f=\sum_{t \geq 0} f_{t}$, where each $f_{t}$ is an eigenfunction associated with the eigenvalue $\lambda_{t}$ of the Laplacian $\Delta$ of $M$. Certainly $f_{0}$ is a constant and the sequence is convergent in $L^{2}$-sense.

Now if $M$ is a submanifold in the Euclidean space $\boldsymbol{R}^{m}$, one has its position vector $x=\left(x_{1}, \cdots, x_{m}\right)$. So by regarding the spectral decomposition of each $x_{i}$ one gets the spectral decomposition of $x$ (see (1.1)). If such a spectral decomposition only involves a finite number of nonzero eigenvalues, say $k$, then the submanifold is said to be of $k$-type (see Section 1 or [5]). From this point of view the easiest spectral behavior corresponds to the submanifolds of 1-type which are characterized, according to a well known result due to Takahashi [12], as minimal submanifolds in some hypersphere of $\boldsymbol{R}^{m}$ whose center and radius are completely determined from the center of mass of $M$ into $\boldsymbol{R}^{m}$ and the associated eigenvalue giving the 1-type character, respectively. Therefore if one wants to study spectral geometry of minimal submanifolds in the sphere, then it seems reasonable to look for the spectral behavior of the products of coordinate functions, $x_{i} \cdot x_{j}$, and then to deal with a very special case of the following problem: What is the eigenvalue behavior of the products of eigenfunctions?

In this case one can organize the product of coordinate functions to give a new isometric immersion in the Euclidean space of symmetric matrices over $\boldsymbol{R}$, this is nothing but the composition of the first isometric immersion with the second standard immersion of the sphere in the Euclidean space according to the description given by Sakamoto [11], and then one can study its type number. This idea was used by Ros [10] to give a characterization for minimal submanifolds in the sphere for which the spectral behaviors of $x_{i} \cdot x_{j}$ involve exactly two different eigenvalues.

* Partially supported by a grant from the Comite Conjunto Hispano-Norte-Americano para la cooperación Cientifica y Tecnológica, Spain. 
One of the authors and Chen [2] (see also [3] for a more general case) also exploited this idea to prove that a Clifford torus in $S^{3}$ and the Veronese surface in $S^{4}$ are the only minimal surfaces in $S^{m}$ which are of 2-type in the space of symmetric matrices over $\boldsymbol{R}$. To be more precise, if $V_{t}$ denotes the eigenspace associated with $\lambda_{t}$ then

$$
x_{i} \cdot x_{j} \in V+V_{t_{1}}+V_{t_{2}}
$$

if and only if either

(1) $t_{1}=2, t_{2}=3$ and the surface is the Clifford torus in $S^{3}$ or

(2) $t_{1}=1, t_{2}=2$ and the surface is the Veronese one in $S^{4}$.

Of course, $x_{i} \cdot x_{j} \in V_{0}+V_{t}$ if and only if $t=2$ and the submanifold is totally geodesic in the sphere.

In this paper once more we exploit this idea to get the following step, so our Theorem 1 can be established as follows: Given a minimal surface in the sphere $S^{n}$ with coordinate functions $x_{i}$ in $\boldsymbol{R}^{n+1}$. Then

$$
x_{i} \cdot x_{j} \in V+V_{t_{1}}+V_{t_{2}}+V_{t_{3}}
$$

if and only if either

(1) $t_{1}=1, t_{2}=2, t_{3}=3$ and the surface is the equilateral tor usin $S^{5}$ or

(2) $t_{1}=2, t_{2}=4, t_{3}=6$ and the surface is the Veronese one in $S^{6}$.

We also take advantage of this method to derive some eigenvalue inequalities for minimal surfaces in the sphere involving the first three eigenvalues. The corresponding inequalities parallel the model (0.1) (see [9] and [10]) cannot give a characterization for the Clifford torus among all minimal surfaces in the sphere, because they only involve the first two eigenvalues. But they give a characterization for the totally geodesic surface and the Veronese surface (in $S^{4}$ ) in terms of intrinsic invariants. Therefore we shall obtain a general inequality (Theorem 2) involving an extrinsic invariant and $\lambda_{1}, \lambda_{2}, \lambda_{3}$ which allows us to give a characterization for totally geodesic surface, Clifford torus, Veronese surface in $S^{4}$ and equilateral torus, among all minimal surfaces in the sphere. Then we regard special properties of these four surfaces to get easier eigenvalue inequalities giving characterizations of some of them. Namely: (1) We observe the isotropy to have one, giving a characterization for totally geodesic surface, Veronese surface in $S^{4}$ and equilateral torus. (2) We regard the parallelism of the second fundamental form, to get one which characterizes the totally geodesic surface, the Clifford torus and the Veronese surface in $S^{4}$. (3) We look for codimension one to have one, giving a characterization for totally geodesic surface and Clifford torus. (See Corollaries 1,2 and 3). Notice that while the first one involves an 
extrinsic invariant the other two only involve intrinsic invariants.

This work was done while the authors were visting the Department of Mathematics, Michigan State University. They wish to thank the Department for providing them with nice facilities. They also want to express their thanks to B.Y. Chen for many valuable comments and suggestions.

1. Some preliminaries. Let $M$ be a compact, connected, Riemannian manifold and $\Delta$ its Laplacian acting on smooth functions in $C^{\infty}(M)$. Then $\Delta$ has an infinite sequence of eigenvalues: $0=\lambda_{0}<\lambda_{1}<\cdots<\lambda_{k}<\cdots \uparrow$ (the spectrum of $M$ ). For each $\lambda_{k}$, the associated eigenspace $V_{k}$ is finitedimensional. On $C^{\infty}(M)$, one considers the usual inner product $(f, g)=$ $\int_{M} f \cdot g d V$, then $\sum_{t \geq 0} V_{t}$ is orthogonal and dense in $C^{\infty}(M)$. So for each $f \in C^{\infty}(M)$, one can talk about the spectral decomposition: $f=\sum_{t \geq 0} f_{t}$, $\Delta f_{t}=\lambda_{t} f_{t}$, which is convergent in $L^{2}$-sense. This decomposition can be extended to $\boldsymbol{R}^{m+1}$-valued smooth functions on $M$, in a natural way.

In particular if $x$ is an isometric immersion of $M$ into $\boldsymbol{R}^{m+1}$ (we can identify $x$ with the position vector of $M$ in $\boldsymbol{R}^{m+1}$ ), then we have the following spectral decomposition of $x$ :

$$
x=x_{0}+\sum_{t \geq 1} x_{t}, \quad \Delta x_{t}=\lambda_{t} x_{t}
$$

where $x_{0}$ is a fixed point in $\boldsymbol{R}^{m+1}$ which coincides with the center of mass of $M$ in $\boldsymbol{R}^{m+1}$. If the spectral decomposition (1.1) is finite, then we will say that $x$ (or $M$ ) is of finite type. Namely, we will say that it is of $k$-type if it involves exactly $k$ nonzero $x_{t}$ 's. If $M$ is of $k$-type and $\lambda_{p_{1}}, \cdots, \lambda_{p_{k}}$ are the associated eigenvalues of its Laplacian, then the set of natural numbers $\left\{p_{1}, \cdots, p_{k}\right\}$ will be called the order of $M$ into $\boldsymbol{R}^{m+1}$. Finite type submanifolds of $\boldsymbol{R}^{m+1}$ are characterized by the existence of a nonzero polynomial, say $P(t)$, such that $P(\Delta) H=0$ ( $H$ being the mean curvature vector of $M$ into $\left.\boldsymbol{R}^{m+1}\right)$. Among all these polynomials, there exists a unique monic polynomial, say $Q(t)$, such that $Q(\Delta) H=0$. Furthermore if $k=\operatorname{deg} Q(t)$, then the submanifold is of $k$-type. Such a unique polynomial is given by

$$
Q(t)=t^{k}+c_{1} t^{k-1}+\cdots+c_{k-1} t+c_{k}
$$

where

$$
c_{1}=-\sum_{i=1}^{k} \lambda_{p_{i}}, c_{2}=\sum_{i<j} \lambda_{p_{i}} \lambda_{p_{j}}, \cdots, c_{k}=(-1)^{k} \lambda_{p_{1}} \cdots \lambda_{p_{k}}
$$

and $\lambda_{p_{1}}, \cdots \lambda_{p_{k}}$ are the associated eigenvalues giving the $k$-type character. Consequently, because $M$ is assumed to be compact and $\Delta x=-n H$ ( $n$ 
being the dimension of $M$ ) one can say that $x: M \rightarrow R^{m+1}$ is of $k$-type if and only if

$$
\Delta^{k-1} H+c_{1} \Delta^{k-2} H+\cdots+c_{k-1} H-\frac{c_{k}}{n}\left(x-x_{0}\right)=0 .
$$

This characterization will be used in this paper. (For more details on this concept see [5]).

On $\boldsymbol{R}^{m}$ we consider the inner product $\langle$,$\rangle given by \langle u, v\rangle=u \cdot v^{t}$ for any $u, v \in \boldsymbol{R}^{m}$, where each vector in $\boldsymbol{R}^{m}$ is regarded as a row matrix and $v^{t}$ denotes the transpose of $v$. Let $r>0$. Then the sphere $S^{m-1}(r)=$ $\left\{u \in \boldsymbol{R}^{m} \mid\langle u, u\rangle=r^{2}\right\}$ with the induced metric has constant sectional curvature $1 / r^{2}$. Let $S M(m)=\left\{P \in g l(m, R) \mid P^{t}=P\right\}$ be the space of symmetric $m \times m$ matrices over $\boldsymbol{R}$ endowed with the metric $g(P, Q)=\left(1 /\left(2 r^{2}\right)\right) \operatorname{tr}(P Q)$ for $P, Q \in S M(m)$. Consider the mapping $f: S^{m}(r) \rightarrow S M(m+1)$ defined by $f(u)=u^{t} \cdot u$. Then $f$ is an isometric immersion which is actually the second standard immersion of $S^{m}(r)$. The image $f\left(S^{m}(r)\right)$ is a real projective space which lies fully in an $(m+m(m+1) / 2)$-dimensional linear space of $S M(m+1)$.

For each point $u \in S^{m}(r)$, the normal space of $S^{m}(r)$ in $S M(m+1)$ at $u$ (or more precisely at $f(u)$ ) is given by

$$
T_{u}^{\perp}\left(S^{m}(r)\right)=\{P \in S M(m+1) \mid u \cdot P=\mu u \text { for some } \mu \in \boldsymbol{R}\} .
$$

In particular, we have $f(u) \in T_{u}^{\perp}\left(S^{m}(r)\right)$.

We will denote by $D$ and $\bar{\nabla}$ the Riemannian connections on $S M(m+1)$ and $S^{m}(r)$ respectively. Also $\bar{\sigma}$ and $\bar{A}$ will denote the second fundamental form and the Weingarten map of $f$ respectively. The following properties of $f$ are well known (see for instance [10]):

It has parallel second fundamental form satisfying

$$
\begin{gathered}
g(\bar{\sigma}(X, Y), \bar{\sigma}(V, W)) \\
=\frac{1}{r^{2}}\{2\langle X, Y\rangle\langle V, W\rangle+\langle X, V\rangle\langle Y, W\rangle+\langle X, W\rangle\langle Y, V\rangle\}, \\
\bar{A}_{\bar{\sigma}_{(X, Y)}} V=\frac{1}{r^{2}}\{2\langle X, Y\rangle V+\langle X, V\rangle Y+\langle Y, V\rangle X\}, \\
g(\bar{\sigma}(X, Y), f(u))=-\langle X, Y\rangle, g(\bar{\sigma}(X, Y), I)=0
\end{gathered}
$$

where $X, Y, V, W \in T_{u}\left(S^{m}(r)\right)$ and $I$ is the identity matrix.

$S^{m}(r)$ is immersed by $f$ as a minimal submanifold of a hypersphere of $S M(m+1)$ centered at $\left(r^{2} /(m+1)\right) I$ and with radius $\left(r^{2} m / 2(m+1)\right)^{1 / 2}$.

2. Some examples. In this section we give some examples of compact 
minimal surfaces in $S^{n}(1)$ which are of finite type in the second standard immersion of $S^{n}(1)$ in $S M(n+1)$.

(1) Consider $x: S^{2}(1) \rightarrow S^{3}(1)$ totally geodesic. Then $\varphi=f \cdot x: S^{2}(1) \rightarrow$ $S M(4)$ is of 1-type and its order is $\{2\}$. This is the only minimal surface in $S^{n}(1)$ which is of 1-type in $S M(n+1)$.

(2) Let $x: M \rightarrow S^{3}(1)$ be the Clifford torus. Then $\varphi=f \cdot x: M \rightarrow$ $S M(4)$ is of 2-type and its order is $\{2,3\}$.

(3) Let $x: M \rightarrow S^{4}(1)$ be the Veronese surface. Then $\varphi=f \cdot x: M \rightarrow$ $S M(5)$ is of 2-type and its order is $\{1,2\}$.

(4) We define an isometric immersion $y$ from $\boldsymbol{R}^{2}$ into $S^{\mathrm{b}}(1)$ by

$$
y(\theta, \tau)=\frac{1}{\sqrt{3}}(\cos \theta, \sin \theta, \cos \tau, \sin \tau, \cos (\theta+\tau), \sin (\theta+\tau)) .
$$

The first fundamental form is given by $g=\left(g_{i j}\right)$ with $g_{11}=g_{22}=2 / 3$ and $g_{12}=1 / 3$. Therefore $y$ induces an isometric immersion from $T=\boldsymbol{R}^{2} / \wedge$ into $S^{\mathrm{b}}(1), \wedge$ being the lattice in $R^{2}$ generated by $\{(\sqrt{2} / 2, \sqrt{6} / 6)$; $(0, \sqrt{2} / \sqrt{3})\}$. Such an immersion will be denoted by $x: T \rightarrow S^{\mathrm{s}}(1)$. According to (2.1), the Laplacian of $T$ is given by $\Delta=-2\left(\partial^{2} / \partial \theta^{2}-\partial^{2} / \partial \theta \partial \tau+\right.$ $\left.\partial^{2} / \partial \tau^{2}\right)$ and so $x$ is minimal in $S^{5}(1)$. Namely it is defined in $\boldsymbol{R}^{6}$ by means of eigenfunctions of $\Delta$ associated with the eigenvalue $\lambda_{1}=2$. It is usually called the equilateral flat torus or the generalized Clifford torus of index 2 (see [7], [8] for more details).

The dual lattice of $\wedge$ is given by

$$
\wedge^{*}=\{(\sqrt{2} h-(\sqrt{2} / 2) k ;(\sqrt{3} / \sqrt{2}) k) \mid h, k \in Z\}
$$

and so the spectrum of $T$ is

$$
\left\{2 h^{2}+2 k^{2}-2 h k \mid h, k \in Z\right\}=\{0,2,6,8,14,18, \cdots\} .
$$

It is not difficult to see that the spectral behaviors of the products of the coordinate functions of $x$ are given by

$$
\begin{aligned}
& x_{i}^{2}-\frac{1}{6} \in V_{8} \quad 1 \leqq i \leqq 6 \\
& x_{1} \cdot x_{2} ; x_{3} \cdot x_{4} ; x_{5} \cdot x_{6} \in V_{8} \\
& x_{k} \cdot x_{j} \in V_{2}+V_{6} \text { otherwise }
\end{aligned}
$$

where $V_{2}, V_{8}$ and $V_{8}$ denote the eigenspaces associated with the eigenvalues $\lambda_{1}=2, \lambda_{2}=6$ and $\lambda_{3}=8$ respectively. As a consequence, we can say that $\varphi=f \cdot x: T \rightarrow S M(6)$ is a 3 -type immersion of order $\{1,2,3\}$. Furthermore its center of mass is nothing but $\varphi_{0}=(1 / 6) I_{6}\left(I_{6}\right.$ being the identity matrix of degree six).

(5) Let $x: S^{2}(\sqrt{6}) \rightarrow S^{8}(1)$ be the third standard immersion of the 
2-sphere. We will call it the Veronese surface in $S^{8}(1)$. The coordinate functions of $x$ are eigenfunctions of the Laplacian of $S^{2}(\sqrt{6})$ associated with $\lambda_{3}$ and so they are harmonic homogeneous polynomials of degree three on $\boldsymbol{R}^{3}$ (restricted on $S^{2}(\sqrt{6})$ ). The isometric immersion $\varphi=$ $f \cdot x: S^{2}(\sqrt{6}) \rightarrow S M(7)$ is at least of 3-type (see [2]). Its coordinate functions are homogeneous polynomials of degree six on $\boldsymbol{R}^{3}$ (restricted on $S^{2}(\sqrt{6})$ ) so in their spectral decomposition cannot appear odd degree polynomials and then $\varphi$ is of 3-type and its order is $\{2,4,6\}$. Furthermore it is easy to see that its center of mass in $S M(7)$ is $\varphi_{0}=(1 / 7) I$.

Notice that the Veronese immersion of $S^{2}(\sqrt{3})$ into $S^{4}(1)$ is of 2-type in $S M(5)$ and its order is $\{2,4\}$, but the induced imbedding of $\boldsymbol{R} P^{2}(1 / 3)$ into $S^{4}(1)$ is also of 2-type in $S M(5)$ and its order is $\{1,2\}$. (Here $R P^{2}(1 / 3)$ denotes the real projective plane of curvature $1 / 3)$. This was the second example. However the Veronese surface in $S^{6}(1)$ (example five) is already an imbedding from $S^{2}(\sqrt{6})$ into $S^{8}(1)$.

3. Spherical minimal surfaces regarded in the space of symmetric matrices. Let $x: M \rightarrow S^{n}(1)$ be a minimal isometric immersion of a compact surface into the unit sphere (without loss of generality we will restrict ourselves to the unit sphere in this paper). Consider a local field of orthonormal frames $\left\{E_{1}, E_{2}, \xi_{3}, \cdots \xi_{n}\right\}$ on $S^{n}(1)$ such that restricted to $M$, $E_{1}, E_{2}$ are tangent to $M$. The following convention will be used for the range of indices $i, j, k, l, r=1,2 ; \alpha, \beta, \gamma, \delta=3, \cdots, n$. We will denote by $\nabla$ the Riemannian connection on $M$ and by $\sigma$ and $A$ the second fundamental form and the Weingarten map of $x$ respectively. The Weingarten endomorphism associated with $\xi_{\alpha}$ will be $A_{\alpha}$. We put

$$
\left\langle\sigma\left(E_{i}, E_{j}\right), \xi_{\alpha}\right\rangle=h_{i j}^{\alpha}, \quad\left\langle(\nabla \sigma)\left(E_{i}, E_{j}, E_{k}\right), \xi_{\alpha}\right\rangle=h_{i j k}^{\alpha} .
$$

Therefore, $\sigma\left(E_{i}, E_{j}\right)=\sum_{\alpha} h_{i j}^{\alpha} \xi_{\alpha}$ and $(\nabla \sigma)\left(E_{i}, E_{j}, E_{k}\right)=\sum_{\alpha} h_{i j k}^{\alpha} \xi_{\alpha}$ (where $\nabla \sigma$ denotes the usual covariant derivative of $\sigma$ ).

We define a symmetric tensor $T$ on the normal bundle of $x$ as follows:

$$
T(\xi, \eta)=\operatorname{tr}\left(A_{\xi} \cdot A_{\eta}\right) .
$$

So the following relations are well known

$$
\begin{gathered}
K=1-\frac{|\sigma|^{2}}{2} \\
\sum_{i} A_{\sigma\left(E_{i}, \bar{X}\right)} E_{i}=\frac{|\sigma|^{2}}{2} X \\
-\frac{1}{2} \Delta|\sigma|^{2}=|\nabla \sigma|^{2}+2|\sigma|^{2}-2|\sigma|^{4}+|T|^{2}
\end{gathered}
$$


where $X$ is any local vector field tangent to $M, \Delta$ and $K$ are the Laplacian and the Gaussian curvature of $M$ respectively and $|F|$ is the length of the tensor $F$.

Given a point $p \in M$, let $U M_{p}$ be the unit sphere on $T_{p} M$ (the tangent plane of $M$ at $p)$ and $f: U M_{p} \rightarrow \boldsymbol{R}$ defined by $f(v)=|\sigma(v, v)|^{2}$. We choose $E_{1} \in U M_{p}$ to be the point at which $f$ attains its maximum and let $E_{2} \in U M_{p}$ perpendicular to $E_{1}$. Then one has

$$
A_{\sigma\left(E_{1}, E_{1}\right)} E_{1}=\lambda E_{1}, \quad A_{\sigma\left(E_{1}, E_{2}\right)} E_{1}=\beta E_{2}
$$

where $\lambda=f\left(E_{1}\right)$ and $\lambda \geqq \beta \geqq 0$. Obviously $\lambda=\beta$ if and only if $M$ is isotropic at $p$. Furthermore, from (3.6), one can obtain

$$
\sum_{i, j} A_{\sigma\left(E_{i}, E_{j}\right)}^{2} X=\frac{|T|^{2}}{2} X
$$

for all $X \in T_{p} M$.

Let us consider the associated immersion $\varphi=f \cdot x: M \rightarrow S M(n+1)$. The mean curvature vector $H$ of $\varphi$ is given by

$$
H=\frac{1}{2} \sum \bar{\sigma}\left(E_{i}, E_{i}\right) .
$$

The Laplacian of $H$ was computed in [10] (see also [3] for a more general formula). So one uses (3.4) to obtain

$$
\Delta H=\left(|\sigma|^{2}+6\right) H-\sum_{i, j} \bar{\sigma}\left(\sigma\left(E_{i}, E_{j}\right), \sigma\left(E_{i}, E_{j}\right)\right) .
$$

REMARK 1. The totally geodesic surface in $S^{n}(1)$ is the only minimal surface in $S^{n}(1)$ which is of 1-type in $S M(n+1)$. Also a Clifford torus in $S^{3}(1)$ and the Veronese surface in $S^{4}(1)$ are the only minimal surfaces in $S^{n}(1)$ which are of 2-type in $S M(n+1)$ (see [2] and [3]). In both cases the center of mass of the surface in $S M(n+1)$ coincides with the center of the hypersphere of $S M(n+1)$ in which $S^{n}(1)$ minimally yields. In this sense we will say that the surface is of mass symmetric in such a hypersphere or by means of the second standard immersion $f$ of the sphere

In order to look for minimal surfaces in $S(1)$ which are of 3-type in $S M(n+1)$, we will compute $\Delta^{2} H$ in the next lemma.

LEMMA 1. Let $x: M \rightarrow S^{n}(1)$ be a compact minimal surface in $S^{n}(1)$ and $H$ the mean curvature vector of $\varphi=f \cdot x: M \rightarrow S M(n+1)$. Then 


$$
\begin{aligned}
\Delta^{2} H= & 2 \nabla|\sigma|^{2}+\left(5|\sigma|^{4}+4|\sigma|^{2}-2|\nabla \sigma|^{2}+36\right) H \\
& +\sum_{\alpha, \beta}\left\{2 \sum_{i, j, k} h_{i j k}^{\alpha} h_{i j k}^{\beta}-\left(5|\sigma|^{2}+2\right) T\left(\xi_{\alpha}, \xi_{\beta}\right)\right\} \bar{\sigma}\left(\xi_{\alpha}, \xi_{\beta}\right) \\
& -4 \sum_{l, \alpha}\left\{\sum_{i, j, k, \beta} h_{i j}^{\beta} h_{k l}^{\beta} h_{i j k}^{\alpha}+\left\langle A_{\alpha} E_{l}, \nabla|\sigma|^{2}\right\rangle\right\} \bar{\sigma}\left(E_{l}, \xi_{\alpha}\right)
\end{aligned}
$$

where $\nabla|\sigma|^{2}$ denotes the gradient of $|\sigma|^{2}$.

Proof. Let $p$ be an arbitrary point of $M$ and $\left\{E_{1}, E_{2}\right\}$ any orthonormal basis in $T_{p} M$. We can extend $\left\{E_{1}, E_{2}\right\}$ to a local field of orthonormal frames on $M$, which will also be denoted by $\left\{E_{1}, E_{2}\right\}$, in such a way that $\nabla_{E_{k}} E_{i}=0$ and $\nabla_{E_{k}} \nabla_{E_{k}} E_{i}=0$ at $p$. In order to compute $\Delta^{2} H$ at $p$, we will use the Einstein summation convention for indices and also we will adopt the following easier notation: $\sigma_{i j}=\sigma\left(E_{i}, E_{j}\right) ;(\nabla \sigma)_{i j k}=$ $(\nabla \sigma)\left(E_{i}, E_{j}, E_{k}\right)$ and $\left(\nabla^{2} \sigma\right)_{i j k l}=\left(\nabla^{2} \sigma\right)\left(E_{i}, E_{j}, E_{k}, E_{l}\right)\left(\left(\nabla^{2} \sigma\right)\right.$ being the usual second covariant derivative of $\sigma)$.

Because $f$ has parallel second fundamental form, one uses (1.6) to get

$$
\begin{aligned}
D_{E_{k}} \bar{\sigma}\left(\sigma_{i j}, \sigma_{i j}\right)= & -\bar{A}_{\bar{\sigma}\left(\sigma_{i j}, \sigma_{i j}\right)} E_{k}+2 \bar{\sigma}\left(\bar{\nabla}_{E_{k}} \sigma_{i j}, \sigma_{i j}\right) \\
= & -2|\sigma|^{2} E_{k}-2 \bar{\sigma}\left(A_{\sigma_{i j}} E_{k}, \sigma_{i j}\right)+2 \bar{\sigma}\left((\nabla \sigma)_{i j k}, \sigma_{i j}\right) \\
& +2 \bar{\sigma}\left(\sigma\left(\nabla_{E_{k}} E_{i}, E_{j}\right), \sigma_{i j}\right)+2 \bar{\sigma}\left(\sigma\left(E_{i}, \nabla_{E_{k}} E_{j}\right), \sigma_{i j}\right) .
\end{aligned}
$$

Now we use the minimality of $x$ together with $\overline{\nabla \sigma}=0$ and (1.6) to obtain

$$
\begin{aligned}
& \Delta \bar{\sigma}\left(\sigma_{i j}, \sigma_{i j}\right)=-D_{E_{k}} D_{E_{k}} \bar{\sigma}\left(\sigma_{i j}, \sigma_{i j}\right) \\
& \left.=2 \nabla|\sigma|^{2}+4|\sigma|^{2} H-2 \bar{A}_{\bar{\sigma}\left(A_{\sigma_{i j}} E_{k}, \sigma_{i j}\right)}\right) E_{k}+2 \bar{\sigma}\left(\nabla_{E_{k}} A_{o_{i j}} E_{k}+\sigma\left(E_{k}, A_{\sigma_{i j}} E_{k}\right), \sigma_{i j}\right) \\
& +2 \bar{\sigma}\left(A_{\sigma_{i j}} E_{k},-A_{\sigma_{i j}} E_{k}+(\nabla \sigma)_{i j k}\right)+2 \bar{A}_{\bar{\sigma}_{\left((\nabla \sigma)_{i j k}, \sigma_{i j}\right)} E_{k}} \\
& -2 \bar{\sigma}\left(-A_{(\nabla \sigma)_{i j k}} E_{k}+\left(\nabla^{2} \sigma\right)_{k i j k}, \sigma_{i j}\right)-2 \bar{\sigma}\left((\nabla \sigma)_{i j k},-A_{\sigma_{i j}} E_{k}+(\nabla \sigma)_{i j k}\right) \\
& =2 \nabla|\sigma|^{2}+4|\sigma|^{2} H+4 \bar{\sigma}\left(A_{(\nabla \sigma) i j k} E_{k}, \sigma_{i j}\right) \\
& +2 \bar{\sigma}\left(\sigma\left(E_{k}, A_{\sigma_{i j}} E_{k}\right), \sigma_{i j}\right)-2 \bar{\sigma}\left(A_{\sigma_{i j}} E_{k}, A_{\sigma_{i j}} E_{k}\right) \\
& +4 \bar{\sigma}\left(A_{\sigma_{i j}} E_{k},(\nabla \sigma)_{i j_{k}}\right)+4\left\langle(\nabla \sigma)_{i j_{k}}, \sigma_{i j}\right\rangle E_{k} \\
& -2 \bar{\sigma}\left(\left(\nabla^{2} \sigma\right)_{k i j k}, \sigma_{i j}\right)-2 \bar{\sigma}\left((\nabla \sigma)_{i j k},(\nabla \sigma)_{i j k}\right) \text {. }
\end{aligned}
$$

Now we use the Ricci identity and the minimality of $x$ to write

$$
\sum_{k}\left(\nabla^{2} \sigma\right)_{k i j k}=\left(2-|\sigma|^{2}\right) \sigma_{i j}+2 \sum_{k} \sigma\left(E_{k}, A_{\sigma j k} E_{i}\right)-\sum_{k} \sigma\left(A_{\sigma_{i j}} E_{k}, E_{k}\right) .
$$

Consequently we obtain

$$
\begin{aligned}
\Delta \bar{\sigma}\left(\sigma_{i j}, \sigma_{i j}\right)= & 4 \nabla|\sigma|^{2}+4|\sigma|^{2} H+2\left(|\sigma|^{2}-2\right) \bar{\sigma}\left(\sigma_{i j}, \sigma_{i j}\right) \\
& +4 \bar{\sigma}\left(\sigma\left(E_{k}, A_{\sigma_{i j}} E_{k}\right), \sigma_{i j}\right)-4 \bar{\sigma}\left(\sigma\left(E_{k}, A_{\sigma_{j k}} E_{i}\right), \sigma_{i j}\right) \\
& +4 \bar{\sigma}\left(A_{\sigma_{i j}} E_{k},(\nabla \sigma)_{i j k}\right)-2 \bar{\sigma}\left(A_{\sigma_{i j}} E_{k}, A_{\sigma_{i j}} E_{k}\right) \\
& -2 \bar{\sigma}\left((\nabla \sigma)_{i j k},(\nabla \sigma)_{i j k}\right)+4 \bar{\sigma}\left(A_{(\nabla \sigma) i j k} E_{k}, \sigma_{i j}\right) .
\end{aligned}
$$


Next by means of a straightforward computation involving (3.4), we have

$$
\bar{\sigma}\left(\sigma\left(E_{k}, A_{\sigma_{i j}} E_{k}\right), \sigma_{i j}\right)-\bar{\sigma}\left(\sigma\left(E_{k}, A_{\sigma_{k}} E_{i}\right), \sigma_{i j}\right)=\frac{1}{2}|\sigma|^{2} \bar{\sigma}\left(\sigma_{i j}, \sigma_{i j}\right) .
$$

Similarly, by involving (3.7), we get

$$
\bar{\sigma}\left(A_{\sigma_{i j}} E_{k}, A_{\sigma_{i j}} E_{k}\right)=|T|^{2} H
$$

and finally, it is not difficult to see

$$
2 \bar{\sigma}\left(A_{(\nabla \sigma)_{i j k}} E_{k}, \sigma_{i j}\right)=\bar{\sigma}\left(E_{i}, \sigma\left(E_{i}, \nabla|\sigma|^{2}\right)\right) .
$$

So, the equation (3.11) becomes the following:

$$
\begin{aligned}
\Delta \bar{\sigma}\left(\sigma_{i j}, \sigma_{i j}\right)= & 4 \nabla|\sigma|^{2}+2\left(2|\sigma|^{2}-|T|^{2}\right) H \\
& +4\left(|\sigma|^{2}-1\right) \bar{\sigma}\left(\sigma_{i j}, \sigma_{i j}\right)+2 \bar{\sigma}\left(E_{i}, \sigma\left(E_{i}, \nabla|\sigma|^{2}\right)\right) \\
& +4 \bar{\sigma}\left(A_{\sigma_{i j}} E_{k},(\nabla \sigma)_{i j k}\right)-2 \bar{\sigma}\left((\nabla \sigma)_{i j k},(\nabla \sigma)_{i j k}\right) .
\end{aligned}
$$

On the other hand, we also use a direct computation to get

$$
\Delta\left(|\sigma|^{2} H\right)=\Delta|\sigma|^{2} H+|\sigma|^{2} \Delta H+6 \nabla|\sigma|^{2}-2 \bar{\sigma}\left(E_{i}, \sigma\left(E_{i}, \nabla|\sigma|^{2}\right)\right) .
$$

Therefore (3.10) follows from (3.5), (3.9), (3.12) and (3.13).

The following result gives a characterization for the surfaces described in Section 2.

THEOREM 1. Let $x: M \rightarrow S^{n}(1)$ be a minimal isometric immersion of a compact surface in the sphere, which is assumed to be full. Then the immersion $\varphi=f \cdot x$ is of 3-type if and only if either

(1) $M$ has constant Gaussian curvature $K=1 / 6$ and $x$ is the Veronese surface in $S^{8}(1)$ or

(2) $M$ is flat and $x$ is an equilateral torus in $S^{5}(1)$.

Proof. According to Section 2, we only need to prove the necessary condition. From (1.3), one has

$$
\Delta^{2} H=a \Delta H+b H+c\left(\varphi-\varphi_{0}\right)
$$

where $\varphi_{0}$ is the center of mass of $\varphi$ and $a=\lambda_{p}+\lambda_{q}+\lambda_{r}, b=-\left(\lambda_{p} \lambda_{q}+\right.$ $\left.\lambda_{p} \lambda_{r}+\lambda_{q} \lambda_{r}\right)$ and $c=-\lambda_{p} \lambda_{q} \lambda_{r} / 2\left(\lambda_{p}, \lambda_{q}, \lambda_{r}\right.$ are the three eigenvalues involved in the 3-type condition).

First, we are going to prove that the Gaussian curvature $K$ of $M$ is constant. We apply $g(\varphi,-)$ to (3.14) and use (1.7) and (3.10) to get

$$
-2|\sigma|^{2}-36=-6 a-b+\frac{c}{2}-c g\left(\varphi, \varphi_{0}\right) .
$$

Therefore for any vector field $X$ tangent to $M$, one gets 


$$
-2 g\left(\nabla|\sigma|^{2}, X\right)=-c g\left(X, \varphi_{0}\right) \text {. }
$$

On the other hand we apply $g(X,-)$ to (3.14) and use (3.10) to obtain

$$
2 g\left(\nabla|\sigma|^{2}, X\right)=-c g\left(X, \varphi_{0}\right) \text {. }
$$

Consequently $\nabla|\sigma|^{2}=0$ and so (3.3) gives the constancy for $K$.

So from (1.4), (3.8), (3.9) and (3.10) we see that $\varphi_{0}$ is normal to $S^{n}(1)$. Because $x$ is full one can use an argument similar to that in [10, Theorem 2.3] to prove that $\varphi_{0}=(1 /(n+1)) I$ ( $I$ being the identity matrix in $S M(n+1))$. Then

$$
\Delta^{2} H=a \Delta H+b H+c\left(\varphi-\frac{1}{n+1} I\right) .
$$

We apply $g\left(\bar{\sigma}\left(E_{r}, \xi_{r}\right),-\right)$ to (3.15) and use (1.5), (1.7), (3.8), (3.9) and (3.10) to get

$$
\sum_{i, j, k, \beta} h_{i j}^{\beta} h_{k r}^{\beta} h_{i j k}^{r}=0 \text {. }
$$

Now by choosing an orthonormal basis $\left\{E_{1}, E_{2}\right\}$ at any point $p \in M$ as in (3.6), (3.16) at $p$ becomes

$$
(\lambda-\beta) h_{111}^{r}=(\lambda-\beta) h_{112}^{r}=0
$$

and it holds for all $\gamma$. Consequently, at each point $p \in M$, we have either $(\nabla \sigma)(p)=0$ or $\lambda=\beta$ and so $M$ is isotropic at $p$. If both facts happen at the same point $p$, from (3.5) one gets that $K=1$ or $K=1 / 3$ and so $M$ is totally geodesic or $M$ is the Veronese surface in $S^{4}(1)$ and it is impossible according to Remark 1 . Because we are assuming $M$ to be connected, it must be parallel or isotropic. If parallel, we can use a result of [6] to find out that $M$ is totally geodesic or a Veronese surface in $S^{4}(1)$ or a Clifford torus in $S^{3}(1)$ and so impossible from Remark 1. Therefore, we conclude that $M$ is isotropic and $(\nabla \sigma)(p) \neq 0$ for all $p \in M$. Then the normal bundle $T^{\perp} M$ of $x$ admits an orthogonal bundle splitting, say $T^{\perp} M=\nu^{*} \bigoplus \nu$, where $\nu_{p}^{*}$ is the first normal space at $p$ (which is of dimension 2). Furthermore from the isotropy it is clear that $\operatorname{Im} g(\nabla \sigma)(p) \subset$ $\nu_{p}$ and $\operatorname{dim}(\operatorname{span}\{\operatorname{Im} \nabla \sigma(p)\}) \leqq 2$ for all $p \in M$.

Now we apply $g\left(\bar{\sigma}\left(\xi_{r}, \xi_{\delta}\right),-\right)$ to (3.15) and use (1.5), (1.7), (3.8), (3.9) and (3.10) to obtain

$$
\left(5|\sigma|^{2}+2-a\right) T\left(\xi_{\gamma}, \xi_{\delta}\right)-2 \sum_{i, j, k} h_{i j k}^{\gamma} h_{i j k}^{\delta}=\left(2|\sigma|^{2}+36-6 a-b+c / 2\right)\left\langle\xi_{r}, \xi_{\delta}\right\rangle .
$$

Moreover $2|\sigma|^{2}+36-6 a-b+c / 2$ is nonzero because $(\nabla \sigma)(p) \neq 0$. So if one chooses $\xi_{r} \in \nu_{p}$ which is perpendicular to $\operatorname{Im} g(\nabla \sigma)(p)$, then the formula (3.18) implies that $\xi_{r}=0$. As a consequence $n \leqq 6$. 
On the other hand one can compute the normal curvature to be $K^{\perp}=$ $|\sigma|^{2} / 2$ (see [1] for details), and then from [1, Theorem 3] we have that $K=1 / 6$ and $M$ is the Veronese surface in $S^{6}(1)$ or $K=0, n=5$ and $x$ is one of the immersions described in [7]. But among those immersions only that corresponding to the equilateral torus is isotropic.

4. Some integral inequalities. Let $\varphi: M \rightarrow R^{m}$ be an isometric immersion of a compact surface in the Euclidean space with mean curvature vector $H$. Then we combine (1.1) with $\Delta \varphi=-2 H$, to get

$$
-2 H=\sum_{t \geq 1} \lambda_{t} \varphi_{t} \text { and }-2 \Delta H=\sum_{t \geq 1} \lambda_{t}^{2} \varphi_{t} .
$$

Consequently one has

$$
\begin{gathered}
4 \int_{M}\langle\Delta H, \Delta H\rangle d v=\sum_{t \geqq 1} \lambda_{t}^{4} a_{t} \\
4 \int_{M}\langle\Delta H, H\rangle d v=\sum_{t \geq 1} \lambda_{t}^{3} a_{t} \\
4 \int_{M}\langle H, H\rangle d v=\sum_{t \geqq 1} \lambda_{t}^{2} a_{t} \\
-2 \int_{M}\langle H, \varphi\rangle d v=\sum_{t \geq 1} \lambda_{t} a_{t}
\end{gathered}
$$

where $a_{t}=\int_{M}\left\langle\varphi_{t}, \varphi_{t}\right\rangle d v$.

Now from (4.1), we have

$$
\begin{aligned}
4 \int_{M}\langle\Delta & H, \Delta H\rangle d v-4\left(\lambda_{1}+\lambda_{2}+\lambda_{3}\right) \int_{M}\langle\Delta H, H\rangle d v \\
& +4\left(\lambda_{1} \lambda_{2}+\lambda_{1} \lambda_{3}+\lambda_{2} \lambda_{3}\right) \int_{M}\langle H, H\rangle d v+2 \lambda_{1} \lambda_{2} \lambda_{3} \int_{M}\langle H, \varphi\rangle d v \\
= & \sum_{t} \lambda_{t}\left(\lambda_{t}-\lambda_{1}\right)\left(\lambda_{t}-\lambda_{2}\right)\left(\lambda_{t}-\lambda_{3}\right) a_{t} .
\end{aligned}
$$

Therefore, one obtains:

LEMMA 2. Let $\varphi: M \rightarrow \boldsymbol{R}^{m}$ be an isometric immersion of a compact surface in the Euclidean space with mean curvature vector $H$. If $\lambda_{1}, \lambda_{2}$ and $\lambda_{3}$ are the first three eigenvalues of the Laplacian of $M$, then

$$
\begin{aligned}
4 \int_{M}\langle\Delta H, \Delta H\rangle d v-4\left(\lambda_{1}+\lambda_{2}+\lambda_{3}\right) \int_{M}\langle\Delta H, H\rangle d v \\
\quad+4\left(\lambda_{1} \lambda_{2}+\lambda_{1} \lambda_{3}+\lambda_{2} \lambda_{3}\right) \int_{M}\langle H, H\rangle d v+2 \lambda_{1} \lambda_{2} \lambda_{3} \int_{M}\langle H, \varphi\rangle d v \geqq 0 .
\end{aligned}
$$

Furthermore, the equality holds if and only if either:

(1) $\varphi$ is of 1-type and its order is $\{1\},\{2\}$ or $\{3\}$, or 
(2) $\varphi$ is of 2-type and its order is $\{1,2\},\{1,3\}$ or $\{2,3\}$, or

(3) $\varphi$ is of 3-type and its order is $\{1,2,3\}$.

Now we have the following:

TheOREM 2. Let $M$ be a minimal compact surface in the unit sphere $S^{n}(1)$. Then

$$
\begin{aligned}
& \int_{M}|T|^{2} d v+2 \int_{M} K^{2} d v+\left(\lambda_{1}+\lambda_{2}+\lambda_{3}-16\right) \int_{M} K d v \\
& \quad+\left[14-\left(\lambda_{1}+\lambda_{2}+\lambda_{3}\right)-\frac{\left(\lambda_{1}-6\right)\left(\lambda_{2}-6\right)\left(\lambda_{3}-6\right)}{4}\right] \operatorname{vol}(M) \geqq 0 .
\end{aligned}
$$

Moreover, the equality holds if and only if either

(1) $M$ is totally geodesic, or

(2) $M$ is the Clifford torus in $S^{3}(1)$, or

(3) $M$ is the Veronese surface in $S^{4}(1)$, or

(4) $M$ is the equilateral torus in $S^{5}(1)$.

Proof. Consider the isometric immersion $\varphi: M \rightarrow S M(m+1), \varphi=f \cdot x$, ( $x$ being the immersion of $M$ into $S^{n}(1)$ ). Then its mean curvature vector $H$ satisfies

$$
\begin{aligned}
& \langle H, \varphi\rangle=-1 \\
& \langle H, H\rangle=3 \\
& \langle\Delta H, H\rangle=|\sigma|^{2}+18 \\
& \langle\Delta H, \Delta H\rangle=|\sigma|^{4}+12|\sigma|^{2}+2|T|^{2}+108 .
\end{aligned}
$$

In order to get it, we only need to use (1.7), (3.8), (3.9) and (3.10). Now from (4.2) and (4.4) we have

$$
\begin{aligned}
& -\left(\lambda_{1}-6\right)\left(\lambda_{2}-6\right)\left(\lambda_{3}-6\right) \operatorname{vol}(M)+2 \int_{M}|\sigma|^{4} d v+4 \int_{M}|T|^{2} d v \\
& +2\left(12-\left(\lambda_{1}+\lambda_{2}+\lambda_{3}\right)\right) \int_{M}|\sigma|^{2} d v \geqq 0 .
\end{aligned}
$$

Then (4.3) follows from (3.3) and (4.5). Certainly the four surfaces listed in the statement of the theorem satisfy the equality in (4.3). Conversely if the equality in (4.3) holds, then we apply Lemma 2 to get either:

(1) $\phi$ is of 1-type which automatically implies that $x$ is totally geodesic (in this case the order of $\phi$ is $\{2\}$ ), or

(2) $\varphi$ is of 2-type and so from [2] (see also Remark 1), $x(M)$ is the Clifford torus in $S^{3}(1)$ or $x(M)$ is the Veronese surface in $S^{4}(1)$ (in these cases the order of $\varphi$ is $\{2,3\}$ and $\{1,2\}$ respectively), or

(3) $\phi$ is of 3-type with order $\{1,2,3\}$ and so from Theorem 1 and 
the examples given in Section 2, we know that $x(M)$ is the equilateral torus in $S^{5}(1)$ (recall that the Veronese surface in $S^{8}(1)$ has order $\{2,4,6\}$ in $S M(7))$.

After this general inequality and because the extrinsic behavior of the examples listed in the statement of the last theorem are well known, we can give the following consequences in which we look at special properties, namely: isotropy, parallel second fundamental form and codimension one.

COROLlary 1. Let $M$ be a minimal compact surface in the unit sphere $S^{n}(1)$. Then

$$
\begin{aligned}
2 \int_{M}|T|^{2} d v \geqq[ & \left.\frac{\left(\lambda_{1}-6\right)\left(\lambda_{2}-6\right)\left(\lambda_{3}-6\right)}{4}+\lambda_{1}+\lambda_{2}+\lambda_{3}-12\right] \operatorname{vol}(M) \\
& +\left(12-\left(\lambda_{1}+\lambda_{2}+\lambda_{3}\right)\right) \int_{M} K d v .
\end{aligned}
$$

Moreover the equality holds if and only if either

(1) $M$ is totally geodesic, or

(2) $M$ is the Veronese surface in $S^{4}(1)$, or

(3) $M$ is the equilateral torus in $S^{5}(1)$.

Proof. It is not difficult to see that for any minimal surface in the sphere, we have

$|\sigma|^{4} \leqq 2|T|^{2}$ equality holding if and only if the surface is isotropic. Now, by using (3.3), (4.7) is equivalent to

(4.8) $2 K^{2} \leqq|T|^{2}+4 K-2$ equality holding if and only if the surface is isotropic.

Then (4.6) follows from (4.3) and (4.8). The equality in (4.6) holds if and only if the equality in (4.3) holds and the surface is isotropic.

COROLlary 2. Let $M$ be a minimal compact surface in the unit sphere $S^{n}(1)$. Then

$$
4 \int_{M}(K-1)\left(10 K-18+\lambda_{1}+\lambda_{2}+\lambda_{3}\right) d v \geqq\left(\lambda_{1}-6\right)\left(\lambda_{2}-6\right)\left(\lambda_{3}-6\right) \operatorname{vol}(M)
$$

Furthermore the equality holds if and only if either

(1) $M$ is totally geodesic, or

(2) $M$ is the Clifford torus in $S^{3}(1)$, or

(3) $M$ is the Veronese surface in $S^{4}(1)$.

Proof. From (3.5), we have

$$
\int_{M}|T|^{2} d v \leqq 2 \int_{M}\left(|\sigma|^{4}-|\sigma|^{2}\right) d v
$$


and the equality in (4.10) holds if and only if $x$ has parallel second fundamental form. Now (4.9) follows from (3.3), (4.3) and (4.10). The equality in (4.9) holds if and only if the equality in (4.3) holds and $x$ has parallel second fundamental form.

CoROLlary 3. Let $M$ be a minimal compact surface in the unit sphere $S^{n}(1)$. Then

$$
4 \int_{M}(K-1)\left(6 K-18+\lambda_{1}+\lambda_{2}+\lambda_{3}\right) d v \geqq\left(\lambda_{1}-6\right)\left(\lambda_{2}-6\right)\left(\lambda_{3}-6\right) \operatorname{vol}(M)
$$

Moreover the equality holds if and only if either

(1) $M$ is totally geodesic, or

(2) $M$ is the Clifford torus in $S^{3}(1)$.

The proof follows from an argument similar to that in the above corollary, by using the following fact:

$|T|^{2} \leqq|\sigma|^{4}$ and the equality holds if and only if $x$ has codimension one.

\section{REFERENCES}

[1] A C. AsPenti, Minimal surfaces with constant normal curvature, J. Math. Soc. Japan 36 (1984), 375-386.

[2] M. Barros and B. Y. Chen, Finite type spherical submanifolds, Proc. II Intern. Symp. Diff. Geom., Lecture Notes in Math., 1209 (1986), 73-93, Springer-Verlag, Berlin, Heidelberg, New York, Tokyo.

[3] M. Barros aNd B. Y. Chen, Spherical submanifolds which are of 2-type via the second standard immersion of the sphere, Nagoya Math. J. 108 (1986).

[4] M. Berger, P. Gauduchon and E. Mazet, Le Spectre d'une Variété Riemannienne, Lecture Notes in Math. 194 (1971), Springer-Verlag, Berlin, Heidelberg, New York.

[5] B. Y. CHEN, Total mean curvature and submanifolds of finite type, World Scientific, 1984.

[6] C.S. Hоuн, Pseudo-umbilical surfaces with parallel second fundamental form, Tensor N.S. 26 (1972), 262-266.

[7] K. KenMoTSU, On a parametrization of minimal immersions of $\boldsymbol{R}^{2}$ into $\boldsymbol{S}^{5}$, Tôhoku Math. J. 27 (1975), 83-90.

[8] K. KeNMotsu, On compact minimal surfaces with non-negative Gaussian curvature in a space of constant curvature: II, Tôhoku Math. J. 27 (1975), 291-301.

[9] S. Montiel, A, Ros and F. URbano, Curvature pinching and eigenvalue rigidity for minimal submanifolds, Math. Z. 191 (1986), 537-548.

[10] A. Ros, Eigenvalue inequalities for minimal submanifolds and $P$-manifolds, Math. Z. 187 (1984), 393-404.

[11] K. Sakamoto, Planar geodesic immersions, Tôhoku Math. J. 29 (1977), 25-56.

[12] T. Takahashi, Minimal immersions of Riemannian manifolds, J. Math. Soc. Japan 18 (1966), 380-385,

Departamento de Geometria y Topologia

UNIVERSIDAD DE GRANADA

18071-GRANADA

SPAIN 